\title{
LA CONCEPCIÓN DEL TIEMPO EN ARISTÓTELES ${ }^{1}$
}

\author{
Jorge Vidal Arenas
}

Universidad de Chile. Chile.

\section{EXISTENCIA Y NATURALEZA DEL TIEMPO}

En el libro IV (217b 29) de la Física, Aristóteles comienza el costumbre, los asuntos a los cuales se referirá y los problemas a los que se debe atender; en primer lugar el físico debe plantearse 1) si el tiempo es o no es (problema de la existencia) y en el caso que sea, 2) cuál es su naturaleza (problema de la esencia del tiempo).

En cuanto al primer punto, afirma el estagirita, si consideramos que el tiempo está compuesto de dos partes (y no tres, pues el presente no es una parte) resultaría evidente que el tiempo no existe de modo absoluto, sino solo de manera relativa y oscura. Pues la primera parte (el futuro) será en algún momento pero aún no es, y la segunda (el pasado) en algún momento fue, es decir, dejó de ser. En vistas de esta situación resultaría dudoso hablar de la existencia del tiempo, puesto que aquello que se compone de partes inexistentes difícilmente podría considerarse como algo que participa del ser.

Nota: Los textos en griego correspondientes a Física fueron extraídos, en cada caso, de la edición bilingüe de la UNAM (Aristóteles, 2001); por su parte, los correspondientes a Metafísica fueron tomados de la edición trilingüe preparada por Gredos (Aristóteles, 1990).

${ }^{1}$ El cuerpo central del que se constituye éste texto fue concebido originalmente como parte de un trabajo más extenso realizado en el seminario de Filosofía Medieval a cargo del profesor Claudio Pierantoni (U. de Chile). En éste se llevó a cabo un análisis comparativo entre las concepciones filosóficas del tiempo en Aristóteles y San Agustín. El motivo por el cual dicho trabajo no fue incluido en su totalidad reside, por una parte, en el hecho de que la autoría del resto del trabajo no me corresponde, y por otra, en que tal inclusión excedería los límites de extensión establecidos. Debo mencionar que cualquier error u opinión(es) vertida(s) en ente ensayo, corresponde(n) exclusivamente a su autor y en ningún caso al profesor a cargo del seminario mencionado. 
Aristóteles afirma que para hablar de la existencia de algo divisible en partes deben cumplirse al menos dos condiciones: la primera es que todas o al menos algunas de sus partes deben existir, y la segunda es que estas partes existentes deben ser medida del todo, es decir, deben tener alguna extensión en el continuo al que pertenecen. Si la primera condición, por los motivos ya aducidos, no es cumplida en absoluto por el tiempo, debemos decir que la segunda, si bien no será negada cabalmente, presenta al menos serias dificultades; pues lo único perteneciente al tiempo de lo cual podemos predicar el ser, es precisamente aquello que no podemos considerar como una extensión y por lo tanto como una parte, a saber, el presente -o en palabras de Aristóteles, el 'ahora'-.

En este punto resulta necesario definir exactamente a qué se refiere Aristóteles con 'ahora' ( $v \tilde{v} v)$ y explicar por qué no debe ser considerado como una parte del tiempo. Esto se lleva a cabo a través de la exposición y resolución del dilema de la identidad y alteridad del 'ahora' (cf. Comentario a Fis. IV (218a 8) de Vigo, Alejandro. En Aristóteles, 1995, p.236).

- ¿El «ahora〉 es siempre uno y el mismo? Si consideramos que cada parte o sección del tiempo que tomemos en cuenta es diferente una de otra, estamos diciendo que es imposible que sucedan simultáneamente (a no ser que una breve esté contenida en una más larga). Si extendemos este principio al «ahora〉 nos daremos cuenta que cada 'ahora' es distinto de otro y no pueden ser siempre uno y el mismo, pues no pueden coexistir ya que no representan extensiones de tiempo, las extensiones de tiempo son partes divisibles comprendidas por límites, y el 'ahora' al no estar comprendido, sino que al ser él mismo el límite, resulta ser inextenso e indivisible.

- Si el 'ahora' no puede ser siempre uno y el mismo, entonces ¿es siempre distinto uno de otro? Puesto que los 'ahoras' no coexisten, si atendemos al hecho de que los 'ahora' del pasado no son existentes habiendo existido efectivamente en algún momento, 
debemos reconocer que aquellos 'ahoras' se destruyeron en algún momento; sin embargo, es imposible que aquel 'ahora' se destruya en otro, puesto que aquello supondría un 'ahora' inmediatamente siguiente al que fue destruido y esto no es posible ${ }^{2}$-tal como un punto no le sigue a otro punto en la línea-. Pero tampoco es concebible que el 'ahora' se destruya dentro de sí mismo (es decir, en el mismo instante en el que es) puesto que es contradictorio que algo sea y no sea al mismo tiempo. Entonces, ¿cómo podemos habar de 'ahoras' distintos unos de otros si no podemos decir que existen aquellos que no están en tiempo presente?

La solución a este importante dilema es entregada por Aristóteles durante el desarrollo posterior de su tratado, sin embargo, para no perder el hilo conductor de esta exposición la examinaremos a continuación.

De igual manera que el movimiento siempre es distinto en el sentido de que cada parte o etapa del movimiento es distinta una de otra, el tiempo también es distinto porque cada lapso específico no se identifica con otro. Ahora, si consideramos el tiempo en su totalidad, podemos decir que es siempre igual, en la medida que los distintos movimientos simultáneos tienen lugar en un único mundo, pues son afectados por un único y mismo tiempo. Tomando en consideración lo anterior, podemos decir que el 'ahora' en cierto sentido es siempre uno y el mismo y en otro es siempre distinto; en cuanto es considerado como 'ser ahora', es decir, lo que sucede en cada instante transcurrido en el tiempo, es siempre diferente (cada 'ahora' posee un ser distinto de otro); Sin embargo, cuando es considerado "como aquello siendo lo cual en cada caso es el ahora"3 (Aristóteles, 1995b, p.89), es decir, como sustrato de los distintos 'ser ahora', es siempre el mismo.

Para asegurar la comprensión del doble sentido en el que debe ser entendido el 'ahora', Aristóteles decide reforzar la idea estableciendo

2 Dado que el tiempo es un continuo, la suposición de que un 'ahora' le sigue a otro es imposible puesto que entre dos 'ahoras', por muy cercanos que sean, siempre existirán infinitos 'ahoras'.

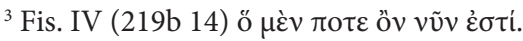


una analogía entre el 'ahora' y el 'móvil' (219b15 - 220a), dicha analogía es, en resumidas cuentas, expuesta de la manera siguiente: el 'ahora' es al tiempo, lo que el 'móvil' es al movimiento. Esto, claro está, en el sentido en que el 'móvil' permanece siendo el mismo como sustrato del movimiento, pero se constituye siempre como algo distinto en cada fase del movimiento. La manera más clara en la que podemos visualizar esto es atendiendo en especial al movimiento locativo o de traslación, pues el 'móvil', por ejemplo una flecha, sigue siendo siempre una y la misma mientras va en el aire, pero es distinta en cada instante que consideremos, en el sentido que ocupa una porción distinta de espacio en cada momento ${ }^{4}$.

\section{Definición del Tiempo}

No podemos entregar una respuesta cabal y definitiva al problema de la existencia del tiempo, sin embargo, la existencia del 'ahora' y sus modos de ser, nos proporcionan importantes elementos para dar paso a la cuestión de la naturaleza del tiempo. Como es de costumbre en nuestro filósofo, el primer paso al respecto lo constituye un examen acerca de las opiniones que han dejado sus predecesores en torno al tema, con esta exposición, pese a lo breve y simplificada que resulta ser, Aristóteles logra introducir los primeros elementos para una definición propia del tiempo.

Las dos consideraciones anteriores acerca del tiempo que tiene en cuenta son las siguientes: 1) El tiempo es el movimiento del todo (o de la esfera celeste) y 2) el tiempo es la esfera misma.

- En primer lugar Aristóteles rechaza la identificación del tiempo con el movimiento de la esfera (circunvolución), puesto que si tomamos solo una parte del movimiento de la esfera y no la circunvolución completa, aun podemos hablar de tiempo; además, el caso hipotético de la existencia de otros mundos supondría la existencia de tiempos distintos, puesto que cada mundo (esfera

\footnotetext{
${ }^{4}$ Ver ejemplo sofistico ofrecido de en 219b 20-23. ("ser Corisco en el Liceo" y "ser Corisco en el ágora").
} 
celeste) tendría su propio movimiento de circunvolución. Pero la existencia de distintos tiempos simultáneos es imposible (en contraposición a la existencia de movimientos simultáneos), pues todos los mundos simultáneos posibles, estarían circunscritos siempre a uno y el mismo tiempo, aun cuando la velocidad de ellos difiera, pues la velocidad no es más que la cantidad de movimiento en un determinado tiempo.

- Finalmente en cuanto a la consideración del tiempo como la esfera misma, la cual Aristóteles desestima como ingenua, se debe al hecho de pensar que, puesto que todas las cosas suceden o están en el tiempo, éstas suceden o están también en la esfera del todo (se trata de un equívoco en cuanto a la expresión "estar en").

Queda manifiesto que entre tiempo y movimiento existe algún tipo de relación, pero está claro que esta relación no es de identificación pues mientras el movimiento solo se da en aquello que cambia, el tiempo se da en todas las cosas, y mientras el movimiento puede variar su velocidad, el tiempo no puede hacerlo, puesto que la velocidad de las cosas que cambian se miden en función del tiempo en que transcurren, pero el tiempo no puede medirse en función de sí mismo. "Es evidente, por tanto, que el tiempo no es movimiento" (Aristóteles, 1995b, p.86).

El tiempo no es movimiento, pero debemos reconocer que no podemos hablar de tiempo sin cambio. Pues cuando en nuestra alma no cambia nada o no advertimos que cambie algo, tampoco advertimos el pasar del tiempo. Dicho de otro modo, el tiempo solo existe para nosotros, en tanto que el alma capte cambio o movimiento. Cada vez que percibimos movimiento percibimos tiempo, es por ello que debemos indagar qué es el tiempo en relación al movimiento.

En primer lugar sabemos que cuando acontece un movimiento, éste se da desde algo hacia algo, es decir se da en un 'continuo'. Y para Aristóteles el 'continuo' por antonomasia es el espacio o 'magnitud'

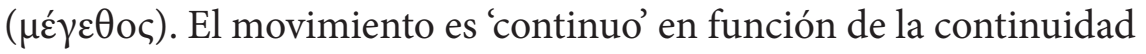


de la magnitud, y el tiempo es 'continuo' en función de la continuidad del movimiento. De esta manera lo anterior y lo posterior que se da primeramente en la magnitud, da origen a la 'antero-posterioridad' del movimiento y éste a su vez da lugar a lo anterior y lo posterior en el tiempo. De esta manera podemos decir que tenemos conocimiento del tiempo cuando podemos determinar un movimiento según lo anterior y lo posterior en él, es decir cuando el alma logra distinguir dos límites o 'ahoras' reconociendo que son distintos entre sí y de lo contenido entre ellos. "Pues esto es el tiempo: número del movimiento según lo anterior y lo posterior"5 (Aristóteles, 1995, p.88). A continuación Aristóteles afirma que existen dos sentidos en los cuales pueden ser entendidos el concepto de 'número': 1) como lo 'numerado o numerable', o 2) como 'aquello a través de lo cual se numera'. De los cuales es el primer sentido es el que se adecúa a su definición del tiempo.

De esta manera, cuando se dice que el tiempo es el 'número' del movimiento según lo anterior y lo posterior, lo que se está numerando no es el movimiento en sí o los 'ahoras-limites' (o una supuesta sucesión de 'ahoras' comprendidos entre los límites) sino que se numera la magnitud o amplitud del movimiento comprendido entre los 'ahoras' (anterior y posterior). Por lo tanto no podemos identificar tiempo y movimiento de manera absoluta, sino solo en tanto que el movimiento comporta número, es decir, en tanto que es numerado. Un indicio de esto, según Aristóteles, sería el siguiente:

Si lo mayor y lo menor (Mym) se mide por medio del número $(\mathrm{N})$, y si un movimiento mayor o menor (Mym) se mide por el tiempo $(\mathrm{T})^{6}$, entonces el tiempo $(\mathrm{T})$ es una especie de número $(\mathrm{N})$. Silogísticamente:

$$
\begin{gathered}
\text { Todo lo Mym es (medido por) } \mathrm{N} \\
\text { Algún Mym es (medido por) } \mathrm{T} \\
\text { El Tiempo es Número }
\end{gathered}
$$

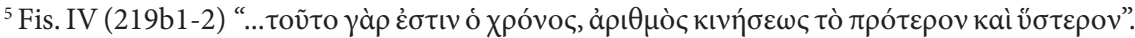
${ }^{6}$ En efecto, un movimiento mayor corresponde a lo que se mueve mucho en poco tiempo y uno menor a lo que se mueve poco en mucho tiempo.
} 
Para finalizar, debemos decir que la analogía anteriormente apuntada entre el 'ahora' y el 'móvil' no se agota en el simple hecho de que ambos comparten la característica de ser en un sentido la misma cosa y en otro siempre algo diferente ${ }^{7}$, sino que también comparten la característica de ser una especie de clave de acceso al conocimiento del 'continuo' al que pertenecen; Por una parte podemos decir que tenemos conocimiento del movimiento solo por medio del 'móvil', y por otra, -si atendemos a la definición de tiempo ya formulada, es decir, "número del movimiento según lo anterior y lo posterior" e identificamos que lo 'anterior' y lo 'posterior' se refiere a dos cortes o 'ahoras' en el tiempo- entonces podemos decir que también tenemos conocimiento del tiempo a través del 'ahora's.

Tal como ya habíamos adelantado, las posteriores partes de éste trabajo se encargarán de abordar las implicancias derivadas de las relaciones tiempo/alma y tiempo/mundo. Para esto, resulta interesante tomar los conceptos (aunque no los análisis) elaborados por Paul Ricoeur en Tiempo y Narración en torno a los dos modos en los que se presenta nuestro problema (Cf. Ricoeur, 2003). ${ }^{\mathrm{P}}$ or una parte, podemos reconocer una aproximación al análisis del tiempo desde una perspectiva que podríamos llamar psicológica, en la cual la pregunta principal a responder sería aquella que indaga acerca de la relación existente entre tiempo y alma. Y por otra parte, nos enfrentamos a una perspectiva que podríamos llamar cosmológica, la cual tendría por objetivo responder a la pregunta por el tiempo y su relación con el mundo. Con esto, pretendemos demostrar que si bien, la medición del movimiento por parte del alma se constituye como la instancia en la cual la existencia del tiempo cobra algún sentido, esta relación no es en modo alguno la condición de posibilidad de manera absoluta de la existencia del tiempo. Pues, a pesar de que los argumentos ofrecidos por el estagirita se encargan de borrar desde

\footnotetext{
${ }^{7}$ Esto es así a pesar de que la relación de identidad y alteridad entre el 'ahora' y el 'móvil' sea asimétrica. (Para este punto Véase: Baño, 2006, n. 11).

${ }^{8}$ Para más detalles acerca de las implicancias del pasaje que va desde $219 \mathrm{~b} 15$ hasta 220a 26, véase, El problema de la analogía entre móvil y 'ahora' en Baño, 2006.
} 
un principio toda distinción nítida entre lo que es la existencia del tiempo y lo que es su percepción (Cf. Vigo, 2002 p.145), y que incluso la misma definición de tiempo que nuestro filósofo entrega de manera definitiva implica la existencia del alma, sostendremos que una lectura atenta del tratado en cuestión nos permitirá esbozar una eventual concepción del tiempo independiente de su relación con el alma (cosmología del tiempo), pues aún sin alma podemos hablar

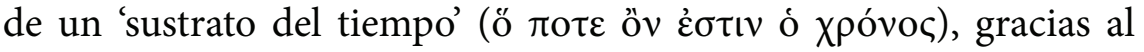
cual nos es posible estudiar la relación tiempo/mundo. En lo que sigue, veremos cuál es la importancia y la función que cumple el 'Motor inmóvil' en relación a los presupuestos cosmológicos que fundan la noción de temporalidad en Aristóteles, pero primero nos encargaremos de cerrar el tema de la relación tiempo/alma, abierto por la misma definición aristotélica del tiempo.

\section{PSICOLOGÍA DEL TIEMPO}

Con el objetivo de esclarecer la función que cumple el alma -según Aristóteles- en relación con el tiempo, nos resulta útil recordar lo mencionado anteriormente respecto de las relaciones entre tiempo y movimiento. Si bien es cierto que Aristóteles no identifica tiempo y movimiento sin más, podemos advertir que existe una relación directa entre estos dos 'continuos' (Cf. Fis. IV 218b18-219a24), pues, como afirma el estagirita, conocemos el tiempo a través del movimiento y el movimiento a través del tiempo (Cf. Fis. IV, 220a15-19); esta relación podemos identificarla de manera clara apelando a su propia definición de tiempo. "Pues esto es el tiempo: número del movimiento según lo anterior y lo posterior"9 (Aristóteles, 1995b. p. 88).

Tras establecer que 'número' puede ser entendido en dos sentidos distintos, a saber, como número numerado (lo que se numera) y como número numerante (a través de lo cual se numera), Aristóteles afirma que en su definición opera claramente el sentido de número numerado, es decir lo que se 'mide'.

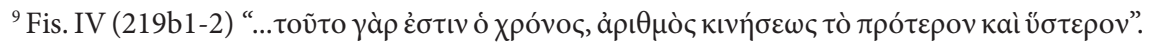


Ahora, esta medición debe ser llevada a cabo obviamente por un agente numerador; y es aquí donde interviene el alma dentro de la concepción aristotélica del tiempo. Es el alma, o su intelecto, quien al discernir dos 'ahoras' distintos en el tiempo, es decir lo anterior y lo posterior en el movimiento, y establecer que estos cortes son también distintos de lo contenido entre ellos, da lugar al tiempo entendido como número del movimiento.

La pregunta que surge inmediatamente es esta: ¿Es posible que tras haber definido el tiempo como número del movimiento, podamos concebirlo como algo que sea capaz de existencia independiente del alma?; La cuestión es planteada por Aristóteles de la siguiente manera:

¿Existiría o no el tiempo si no existiese el alma? Porque si no pudiese haber alguien que numere tampoco podría haber algo que fuese numerado, y en consecuencia no podría existir ningún número, pues un número es o lo numerado o lo numerable. ${ }^{10}$ (Aristóteles, 1995a, p.287).

La respuesta de Aristóteles a esta aparente complicación resulta ser bastante escueta, y se basa sustancialmente en la apelación a su definición entregada. Puesto que el tiempo es número del movimiento, resulta necesaria la existencia de un agente capaz de numerar este movimiento para que el tiempo pueda existir de manera efectiva. Por lo tanto "resulta imposible la existencia del tiempo sin la existencia del alma, [a menos que sea aquello por lo cual existe el tiempo], como sería el caso si existiera el movimiento sin que exista el alma"11 (Aristóteles, 1995a); En efecto, si hacemos abstracción del tiempo

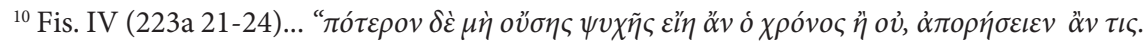

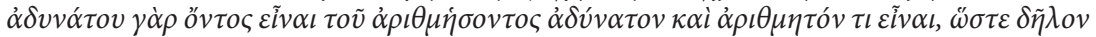

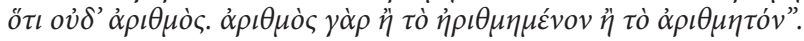

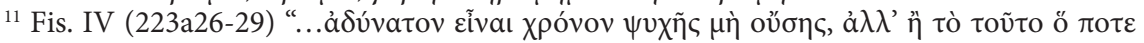

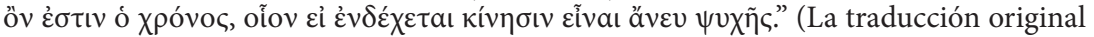
de este pasaje realizada por Guillermo de Echandía (Aristóteles, 1995a) fue modificada ligeramente en la frase que arriba destacamos en 'negrita'. La traducción de la mentada frase fue sustituida por la de Alejandro Vigo (Aristóteles, 1995b), que creemos, recoge mucho mejor el sentido de 'sustrato' proporcionado por la formula «ö $\pi 0 \tau \varepsilon$ ö $\Downarrow$ ».
} 
como numeración del movimiento, y atendemos a aquello por lo cual el tiempo es número, queda algo que no es tiempo propiamente tal, sino únicamente su substrato (Cf. Vigo, 2006, p. 53), es decir, el movimiento que potencialmente puede comportar número.

Como ha señalado oportunamente Alejandro Vigo (Cf. Ibíd. p. 52), el pasaje que va desde 223 a21 hasta 223a29, ha presentado desde antiguo varias complicaciones para su interpretación, esto se debe en gran parte a la lectura que se le ha aplicado al pasaje desde la óptica de la modernidad, involucrando las categorías que aluden al problema de la esencia del conocimiento, a saber, realismo e idealismo. Vigo afirma que el problema esencial pasa justamente por el hecho de que el tratamiento aristotélico en torno a la cuestión del alma en relación al movimiento - y en definitiva, la cuestión del alma en relación al mundo en general- no se deja encasillar bajo ninguna de estas categorías.

En efecto, una interpretación idealista se disiparía de inmediato al considerar que lo que Aristóteles afirma no es que el tiempo se dé dentro del alma o que sea ésta quien lo constituya, sino que solo se sostiene que el tiempo, al ser número del movimiento, se encuentra en estrecha relación con aquello que lleva a cabo la numeración.

Por otra parte, una interpretación realista la cual supondría la existencia real de los objetos de conocimiento independientemente del sujeto cognoscente, podría tomar como base lo dicho por Aristóteles en Met. IV 5, 1010b30-1011a2:

Y, en suma, si sólo existe lo sensible, no existiría nada si no existieran los seres animados, pues no habría sensación. Que, en efecto, no existirían lo sensible ni las sensaciones sin duda es verdad (pues esto es una afección del que siente); pero que

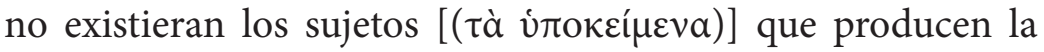
sensación, incluso sin sensación, es imposible. La sensación, en efecto, no es, ciertamente, sensación de sí misma, sino que hay 
también, además de la sensación, otra cosa, que necesariamente es anterior a la sensación... ${ }^{12}$ (Aristóteles, 1990, p.194).

Pues, al atender a aquella otra cosa que es anterior a la sensación ( $\tau \grave{a}$

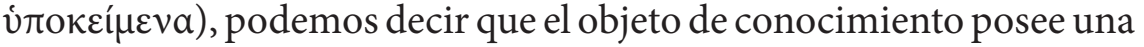
existencia independiente de las determinaciones que el alma pueda captar en ella. Sin embargo, resulta necesario hacer la distinción clara entre lo que sería un objeto primario de percepción (p.ej. El color, el sabor, y en definitiva cualquier tipo de determinación perteneciente a la materia en tanto que sensible) y lo que sería el substrato de dichas determinaciones; pues, mientras el primero no posee existencia actual independiente del acto mismo de la percepción, el segundo puede existir con independencia de la actividad perceptiva del alma puesto que solo es aquello por lo cual tiene lugar lo sensible (Cf. Vigo, 2006, p. 55).

Dicho lo anterior, debemos reconocer que el tiempo no es un objeto sustancial, es decir, no es un substrato independiente del alma, sino que es una determinación de aquellos objetos sustanciales que comportan movimiento. Es decir, su existencia, en tanto que afección del movimiento, resulta estar íntimamente ligada con la percepción de éste llevada a cabo por el alma. Precisamente éste es el sentido de las siguientes palabras. "Los [momentos de] antes y después se dan en el cambio, pero el tiempo se da en tanto que estos [momentos] se pueden contar"13 (Aristóteles, 2001, p. 109).

En consecuencia, podemos afirmar con Aristóteles que el tiempo no consiste en una mera inspección del alma ni tampoco en una mera determinación del movimiento, sino que más bien resulta ser

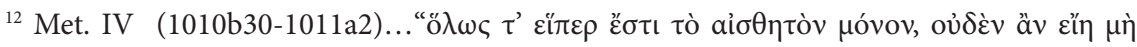

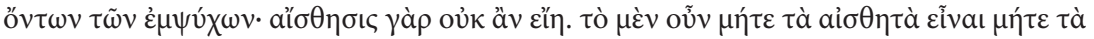

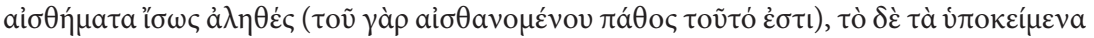

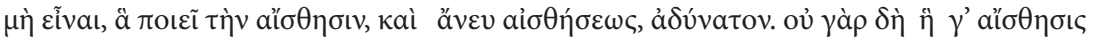

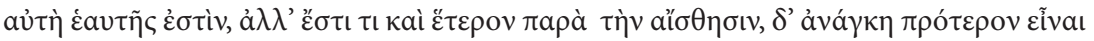
$\tau \tilde{\eta} \varsigma$ aio $\theta \dot{\sigma} \sigma \varepsilon \omega \varsigma "$.

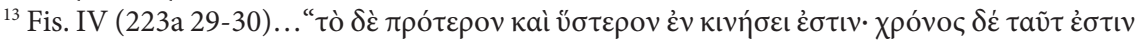

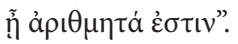


Jorge Vidal Arenas: La concepción del tiempo en Aristóteles.

la instancia en donde confluyen en una y la misma actualización, la potencia que el alma tiene para numerar con la potencia que el movimiento tiene para ser numerado

Lo que Aristóteles afirma no consiste en una negación absoluta de la existencia del tiempo con independencia del alma, pues el tiempo es numero 'numerado' del movimiento, y lo numerado también puede ser entendido como 'numerable' (Fis. 219b7-8) (es decir la potencia de ser numerado), con esta precisión podemos decir que para hablar de tiempo no es necesario que dicha numeración se dé en acto, puesto que podemos suponerla actual. (Cf. Vigo, 2002, pp.143144) Es así, gracias a esta última consideración, que nos es posible hablar de una cosmología del tiempo.

\section{COSMOLOGÍA DEL TIEMPO}

Tanto para Aristóteles como para la mentalidad griega en general, el mundo es ingénito e incorruptible, no hay lugar para una creación desde la nada ni para el paso del ser al no-ser absoluto del mundo. Sin embargo es en la particularidad de los argumentos donde las diferencias se hacen patentes en este terreno, pues mientras que para Platón el mundo resulta ser -según el mito del Timeo- formado y configurado por un Demiurgo que obra sobre una materia prima preexistente en un 'tiempo caótico' e indefinido ${ }^{14}$, Aristóteles afirma que el mundo, refiriéndose a todos sus componentes y afecciones, tales como el espacio, el movimiento y el tiempo, son coeternos a una realidad eterna y primera, que es tal porque es causa primera del movimiento.

Dicho esto, resulta necesario analizar los presupuestos que conducen finalmente a la necesidad de afirmar la existencia de un

${ }^{14}$ No profundizaremos aquí en el extenso debate acerca del verdadero sentido que tienen las

líneas expuestas en el Timeo: si acaso se trata solo de una serie de expresiones alegóricas para representar un orden causal-lógico del mundo, o si se trata de una exposición literal en donde el mundo, tal como lo conocemos, es concebido como venido a la existencia después de ser ordenado -cronológicamente hablando- por un Demiurgo. Basta con decir que el mismo Aristóteles se inscribió en esta segunda línea interpretativa. Para más detalles al respecto, véase el breve estudio introductorio a la traducción del Timeo en Platón, 2007, pp.127 ss. 
Motor inmóvil, que si bien, no se involucra de manera directa con el mundo, podríamos decir que gracias a él éste último subsiste.

Para Aristóteles resulta absurdo que el mundo sea generado, es algo que la lógica no puede permitir. La demostración más clara de esta doctrina se da en Física VIII, en la cual, si bien Aristóteles se refiere de manera específica a la eternidad del movimiento, es posible extender dicha argumentación a la eternidad tanto del tiempo como del mundo material (o de la magnitud espacial) ${ }^{15}$. Esto lo podemos hacer en virtud de la característica que comparten -tanto espacio, movimiento y magnitud- de ser continuos: Lo continuo es aquello que posee un antes y un después, "ahora bien, el antes y el después son ante todo atributos de un lugar, en virtud de su posición relativa. Y puesto que en la magnitud hay un antes y un después, también en el movimiento tiene que haber un antes y un después, por analogía con la magnitud. Pero también en el tiempo hay un antes y un después pues el tiempo sigue siempre al movimiento"16 (Aristóteles, 1995a, p. 270). En efecto, mientras el tiempo es algo del movimiento (es más, no podemos pensar el uno sin el otro (Cf. 218b20-219a1)), el movimiento solo se puede dar a través de la magnitud espacial, puesto que el movimiento es movimiento de algo y no de sí mismo. Es decir, se da con necesidad de un sujeto que soporte el cambio y sirva de sustrato. Por lo tanto, cuando afirmamos la eternidad del movimiento, también lo hacemos respecto del tiempo y el mundo.

Aristóteles inicia el razonamiento de esta manera:

¿Alguna vez fue engendrado el movimiento, no habiendo existido antes, $y$ ha de ser destruido alguna vez, de manera que

15 A pesar de que nuestra exégesis acerca de la infinitud del tiempo y del mundo la subordinamos aquí a los argumentos entregados en Física VIII a favor de la infinitud del movimiento, no debemos pasar por alto que Aristóteles también argumenta de manera individual la infinitud tanto del tiempo (Fis. IV 222a10-b7; 251b10-29) como del mundo (De Cael. I 279b4-283b24).

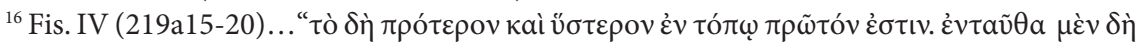

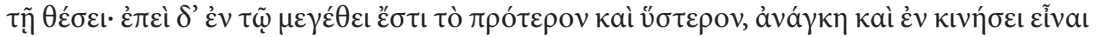

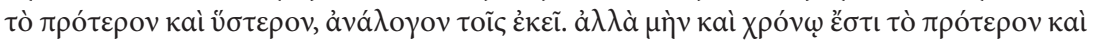

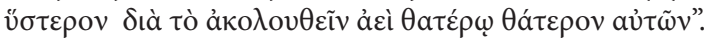


ya nada estará en movimiento? ¿O no fue engendrado ni será destruido, sino que siempre existió y siempre existirá, y esto inmortal e incesante pertenece a la cosas (...)? ${ }^{17(\text { Aristóteles, 1995a, p.423) }}$

Podríamos resumir las opciones entregadas de la siguiente manera: 1) ¿el movimiento (y por lo tanto el mundo) ha sido generado para luego llegar a un término? o 2) ¿éste es eterno, sin generación ni destrucción?

Para responder a la interesante pregunta-que por lo demás, resulta de gran interés para el debate entre paganismo y cristianismo-, Aristóteles se basa sustancialmente en argumentos de tipo lógico-causal.

\section{Eternidad del mundo, del movimiento, y del tiempo}

Tal como observa acertadamente Aristóteles, independientemente de la postura tomada en torno a la cuestión de la finitud o eternidad del mundo, todos, o al menos la gran mayoría de los que han hechos cosmogonías, han aceptado explícita o tácitamente la existencia del movimiento, pues todos han hablado de generación o corrupción, y estos precisamente consisten en formas de cambio. Qué es el movimiento, es algo que ya fue discutido en el tercer libro de la Física (201a9-15), a saber, "la actualidad de la potencia en tanto que es potencia”. Cuando se dice que el movimiento comenzó en algún momento sin antes haber existido, tenemos dos opciones: 1) Que el mundo haya sido generado junto al movimiento o para luego comenzar a moverse, o 2) que el mundo sea eterno y existente desde antes que el movimiento tenga inicio.

1. Si aceptamos que el mundo fue generado para luego iniciar el movimiento, debemos atender al hecho de que esta generación ya constituye un tipo de cambio, pues si algo no existe, y tiene la potencia de existir, al momento de venir a la existencia o de

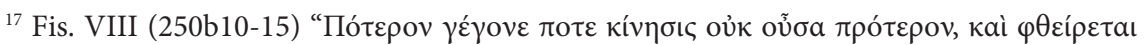

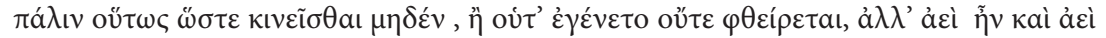

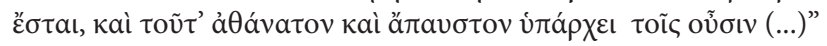


generarse significa que ha actualizado una potencia, y por lo tanto -en función de la definición- es movimiento ${ }^{18}$, y sería anterior al supuesto inicio del movimiento. Además, el solo hecho de decir que el mundo se genera 'junto al movimiento', debe ser rechazado, pues al hablar de generación, se está suponiendo un tiempo preexistente en el cual no existía el mundo ni el movimiento, y puesto que el tiempo solo es concebible cuando hay movimiento (o viceversa) ${ }^{19}$, esta expresión se constituiría bajo un supuesto falso.

2. Ahora bien, suponer que el mundo -siendo eterno- tenga un comienzo del movimiento, es algo absurdo. Pues, si en estas condiciones el movimiento comenzó en algún momento, también debemos suponer que existió un primer móvil y un primer motor, pero aquello que es motor, para poder mover requiere en sí tener movimiento, y este movimiento solo le pudo ser comunicado por otro motor, y así sucesivamente en una serie infinita.

\section{El Motor Inmóvil}

A pesar de negar la generación del movimiento, debemos reconocer que el movimiento, por eterno que sea, debe tener alguna causa primera que no nos lleve al infinito. Pues según el célebre principio de causalidad: "todo lo que está en movimiento tiene que ser movido por algo"20 (Aristóteles, 1995a, p.391). Ya sabemos, según nuestra definición de movimiento, que todo lo que se mueve y por lo tanto que es capaz de producir cambio debe estar en acto, por ejemplo, un cuerpo frío se calienta por la acción de un cuerpo que ya está caliente; Dicho esto, surge una aporía: pareciera que todo lo que está en acto tuvo una potencia (aquello que calentó lo frío no era caliente por sí mismo o caliente eternamente, sino que adquirió el calor por otro cuerpo), pero la potencia no tiene por qué tener un acto, pues lo potencial puede no ser. Y en este sentido, la potencia sería anterior al acto.

\footnotetext{
${ }^{18}$ Para más detalle acerca de la 'ingenerabilidad' e 'incorruptibilidad' del mundo Véase: $D e$ Cael. I 279b4-280a35.

${ }^{19}$ Cf. Física IV (218b21-219a2).

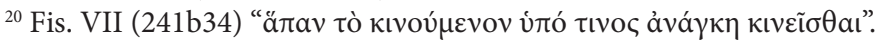


Pero si esto fuera así, no existiría ninguna de las cosas que son, ya que es posible que algo pueda ser, pero no sea. Y si fuera como dicen los teólogos que hacen surgir todo de la noche, o como dicen los filósofos de la naturaleza que «todas las cosas estaban juntas», surgiría la misma imposibilidad. Y es que ¿cómo se habría producido el movimiento de no haber causa alguna en acto ? $^{21}$ (Aristóteles, 1994, p.483)

Si el movimiento, ciertamente, es eterno, necesita de un motor que sea siempre acto (acto puro), puesto que si tuviera potencia alguna, no habría movimiento eterno, pues lo potencial podría no ser (Cf. Met. XII 1071b16-18). Por lo tanto, si nuestra causa primera debe ser un acto eterno (que sustente el movimiento eterno del mundo) y libre de toda potencia, éste debe ser también inmaterial, puesto que solo la materia comporta potencialidad (ya que el movimiento y el cambio solo se dan en ella). Finalmente, nuestra causa primera al exigir ser acto puro, no puede contemplar ni entrar en contacto con otra cosa que no sea su propia perfección, ya que cualquier contacto con el mundo material, lo contaminaría con potencialidad. Una vez despejado todos los atributos que corresponden al primer motor -ser eterno, inmaterial, acto puro e inmóvil ${ }^{22}$ - solo queda la siguiente pregunta: ¿si el Dios aristotélico no es causa del movimiento a modo de causa eficiente -pues no puede entrar en contacto directo con el mundo-, de qué manera es causa eterna del movimiento?

No es un misterio el que una de las diferencias esenciales entre el pensamiento griego y el cristiano, consista en que este último a diferencia del primero, sostenga su pensar cosmológico sobre la

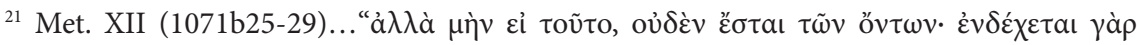

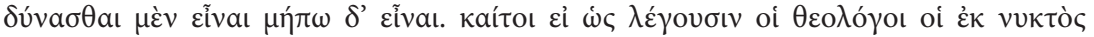

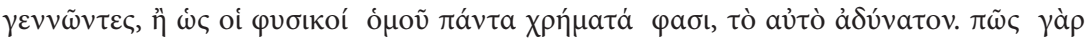

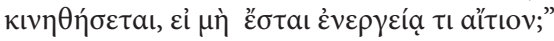

${ }^{22}$ El atributo de inmóvil en realidad se desprende de su característica de ser acto puro, pues, mientras el reposo consiste en la ausencia de movimiento sin negar la posibilidad de moverse, lo inmóvil consiste en aquello que sin estar en movimiento no posee la facultad de hacerlo. 
doctrina 'ex nihilo'. Diferencia que en términos aristotélicos podría retraducirse en la distinción entre el tipo de causa primera que se propone como fundamento de la existencia del mundo.

Mientras que el Dios cristiano es causa del mundo a modo de causa eficiente, puesto que crea el mundo desde la nada tal como un artífice crea desde la materia ${ }^{23}$, el Dios aristotélico es causa del movimiento a modo de causa final, tal como el amado mueve al amante (Cf. Metafísica. XII 7. 1072b2-3) de esta manera el motor inmóvil se libra de todo contacto con el mundo material.

Para Aristóteles, el mundo no tiene ni puede tener una causa eficiente, puesto que tal causa implicaría una cadena de causas hacia el infinito, sin embargo, podemos pensar en una causa primera que no es primera en sentido cronológico si logramos comprender que la eternidad de dicha causa primera es coeterna a la eternidad del tiempo y el mundo.

${ }^{23} \mathrm{Si}$ bien, la analogía es evidentemente insuficiente para expresar el tipo de creación desde la nada, puesto que el artífice efectivamente si crea desde un algo, basta para ejemplificar el tipo de causa que representa el Dios cristiano. 


\section{Referencias bibliográficas}

ARISTÓTELES. (1990). Metafísica de Aristóteles. Edición trilingüe por Valentín García Yebra. Madrid: Gredos.

ARISTÓTELES. (1994). Metafísica. Introducción, traducción y notas de Tomás Calvo Martínez. Madrid: Gredos.

ARISTÓTELES. (1995a). Física. Introducción, traducción y notas de Guillermo R. de Echandía. Madrid: Gredos.

ARISTÓTELES. (1995b). Física: Libros III - IV. Traducción, introducción y comentario por Alejandro G. Vigo. Buenos Aires: Biblos.

ARISTÓTELES.(1996).Acerca delcielo-Meteorológicos. Introducción, traducción y notas de Miguel Candel. Madrid: Gredos.

ARISTÓTELES. (2001). Física, Edición bilingüe. Traducción y notas de Ute Schmidt Osmanczik, Introducción de Antonio Marino López. México D.F.: Universidad Nacional Autónoma de México. ARISTÓTELES. (2003). Física: Libros VII - VIII. Traducción, introducción y comentario por Marcelo D. Boeri. Buenos Aires: Biblos.

BAÑO, PALOMA.. (2006). El 'ahora' aristotélico como límite y como tránsito. Revista Tópicos $\mathrm{N}^{\circ} 30$ bis. Universidad de Chile.

PLATÓN.(2007) Timeo en.Diálogos VI. Traducciones, introducciones y notas por María Ángeles Durán y Francisco Lisi. Madrid: Gredos.

RICOEUR, PAUL. (2003). Tiempo y narración III. Buenos Aires: Siglo XXI editores.

VIGO, ALEJANDRO. (2002). Indiferentismo ontológico y fenomenología en la Física de Aristóteles. Revista Noua tellus, $\mathrm{N}^{\circ}$ 20.2. Universidad autónoma de México.

VIGO, ALEJANDRO. (2006). ¿Está obsoleta la Física de Aristóteles? Revista Estudios Públicos Nº102. 